\title{
Radiological management of COVID-19: structure your diagnostic path to guarantee a safe path
}

\author{
Roberto Stramare ${ }^{1}$ Giovanni Carretta ${ }^{2}$ Alfio Capizzi ${ }^{2} \cdot$ Deris Gianni Boemo ${ }^{2} \cdot$ Cristina Contessa $^{2} \cdot$ Raffaella Motta $^{1}$. \\ Giorgio De Conti ${ }^{3} \cdot$ Francesco Causin $^{4} \cdot$ Chiara Giraudo $^{1}$ (1) Daniele Donato ${ }^{2}$
}

Received: 16 April 2020 / Accepted: 25 May 2020 / Published online: 4 June 2020

(c) Italian Society of Medical Radiology 2020

\begin{abstract}
The current COVID-19 outbreak is requiring a tremendous effort not only regarding the diagnostic and therapeutic approach but also in terms of global management of the delivered care. Hospital administrations had to provide a prompt response to a rapidly evolving emergency characterized by the necessity of giving access to an enormous number of infected patients, guaranteeing appropriate care to patients in need of other types of treatment, and simultaneously preserving the well-being of healthcare providers. To optimize the diagnostic pathway during the current COVID-19 outbreak, the hospital administration of our tertiary center applied a highly structured framework assigning specific tasks to the different units composing the Department of Imaging. In particular, since the beginning of the pandemic, a mobile CT scanner in a truck was rented and became operative for all patients with a confirmed diagnosis of COVID-19 and another CT was assigned for all suspected cases. The success and efficacy of the management applied by our administration is demonstrated by the fact that during the outbreak, the radiological workflow was never interrupted. In fact, despite the national lockdown only a $29.3 \%$ decrease of CT scans occurred compared to the previous year. Moreover, none of the healthcare providers of the Department contracted the infection at work. Thus, according to the experience gained in our center, we recommend to all hospital administrations facing the COVID-19 outbreak to promptly adapt their resources, creating precise and safe pathways for their diagnostic units.
\end{abstract}

Keywords Management $\cdot$ COVID-19 $\cdot$ Radiology $\cdot$ Safety $\cdot$ Emergency

The current COVID-19 outbreak represents a major challenge for all healthcare systems not only regarding the diagnostic and therapeutic processes but also in terms of global management of the delivered care $[1,2]$. Hospital administrations had to provide a prompt response to a rapidly evolving emergency characterized by the necessity of giving access to an enormous number of patients and simultaneously preserving the well-being of the healthcare providers.

Chiara Giraudo

chiara.giraudo@unipd.it

1 Institute of Radiology, Department of Medicine-DIMED, University of Padova, Via Giustiniani 2, 35100 Padova, Italy

2 Department of Directional Hospital Management, Azienda Ospedaliera Di Padova, Padova, Italy

3 Radiology Unit, Azienda Ospedaliera di Padova, Padova, Italy

4 Neuroradiology Unit, Azienda Ospedaliera di Padova, Padova, Italy
Among the units directly involved in the management of the clinical pathways, the diagnostic sections surely required an accurate organization [3].

As demonstrated in the literature, radiological imaging plays a crucial role in the assessment of COVID-19 patients starting from the early phases of the disease but we should not focus only on the contribution of specific techniques or the typical signs of the infection at imaging. In fact, highquality imaging can be provided only if healthcare administrators properly handle the organizational aspect of the emergency. As shown for previous epidemics like SARS, a structured approach to the radiological departments including the implementation of specific devices has a positive impact on the overall management of patients [4].

In particular, during the current pandemic, attention had to be devoted in separating suspected from confirmed COVID-19 patients in need of urgent radiological imaging avoiding any possible contamination or delay in the provided care. Especially regarding the last aspect, molecular tests which are, up to now, the main applied diagnostic 
tool, require some hours to be processed. In this interval of time, the clinical pathway of critical patients should not be interrupted and Emergency Rooms (ER) should not be overcrowded.

Establishing proper pathways can be particularly challenging in large hospitals, as ours, acting usually as referring centers for numerous and various diseases. In fact, our hospital has 1727 beds including 87 in intensive care units (ICU) and offers tertiary care in different fields such as liver and lung transplant, pediatric surgery, and oncology.

The Department of Diagnostic Imaging is composed of one Nuclear Medicine unit, and four radiological units subdivided as follows: one neuroradiology unit, one general radiology unit including the main ER admission, one academic radiology unit including a third level pediatric section, and one general radiology unit located in a nearby building with a separate secondary ER admission (Fig. 1). Each section has one CT scanner. Only the general radiology, located in one of the main buildings, has two CTs but with a shared control room (Fig. 1).

To face this challenge and in particular to optimize the diagnostic pathway during the current COVID-19 outbreak, the hospital administration of our tertiary center applied a highly structured framework assigning specific tasks to the different units composing the Department of Imaging.

Few days after the beginning of the pandemic in the Regione Veneto (i.e., 21st of February 2020), a mobile CT scanner in a truck was rented and became immediately operative for all patients with a confirmed diagnosis of COVID-19. Moreover, the CT of the academic radiological unit (orange in the graph) has been assigned for examining all suspected cases of COVID-19 (i.e., symptomatic or asymptomatic subjects at high risk of infection, waiting for the results of the molecular test and requiring urgent scans).

In case of patients in critical conditions who cannot be transported to the truck, the CT of the academic unit is used and then properly disinfected and locked following the guidelines [5]. All other CTs of the hospital (i.e., green in the graph) are used for non-COVID-19 patients, aiming to avoid any interruption of the routine clinical activity especially regarding surgical, oncological, and pediatric patients who require care that cannot be postponed.

Obviously, all patients who require an urgent $\mathrm{CT}$ scan but cannot be transported to any of the two assigned CTs (i.e., mobile CT for confirmed cases, $\mathrm{CT}$ of the academic unit for suspected patients) are examined in the nearest radiological unit and afterwards the CT room is carefully cleaned and disinfected [5].

The success and efficacy of the management applied by our administration is demonstrated by the fact that during the outbreak, the radiological workflow was never interrupted. In fact, during the first month of the pandemic in the Regione Veneto, in our hospital, 1946 CT scans were performed, 69 of which using the mobile device (i.e., for COVID-19-positive patients), and 539 with the academic scanner (i.e., for suspected COVID-19, COVID19 patients who could have not been transported to the truck, and non-COVID-19 patients referring to the academic unit). Considering that the national lockdown led to a reduction of the deferrable surgical procedures and clinical assessments, and in turn of the prescribed radiological examinations, in our center, in comparison to the same interval of time of the previous year, only a $29.3 \%$ decrease of CT scans occurred.

It certainly has to be noted that all COVID-19 patients are mainly monitored by chest X-rays and our hospital has 11 mobile X-ray devices which can be assigned for infected patients. Nevertheless, in case of clinical conditions requiring CT scans, it is mandatory to assure that these examinations are safely performed [6].

A further sign of the success of the applied method is represented by the evidence that according to the surveillance of healthcare providers belonging to the Department of Diagnostic Imaging, up to now, none contracted the disease. Only one technician of the neuroradiology unit, completely asymptomatic, tested positive for COVID-19 but is believed to have contracted the virus from a household member. $\mathrm{He}$ went immediately to quarantine and none else in his unit was affected.

All healthcare providers of the Department worked in safety, using the recommended protective equipment (i.e., surgical mask and gloves dealing with non-COVID-19 patients; filtering masks, gloves, long sleeved gown, protective shoe covers, and eye protection dealing with suspected or positive COVID-19 patients) [7]. Patients had to wear a mask, the safety distance of at least $2 \mathrm{~m}$ was applied in all waiting rooms, caregivers had limited access, and everyone accessing the Department was screened (e.g., temperature check, anamnesis focused on the main COVID-19 symptoms).

As suggested in the recent article of Mossa-Basha et al., the preparedness of hospitals is essential to face this dramatic situation [8]. According to the experience gained in our center, we recommend to all hospital administrations facing the COVID-19 outbreak to promptly adapt their resources, creating precise and safe pathways for their diagnostic units.

We strongly believe that only a precise organization of the workload, figuring out the designation of devices dedicated not only to confirmed COVID-19 cases but also to suspected patients, will provide a fully beneficial service for the entire healthcare system, assuring the best delivered care to all patients, guaranteeing the safety of the healthcare providers, and optimizing the economic resources. 


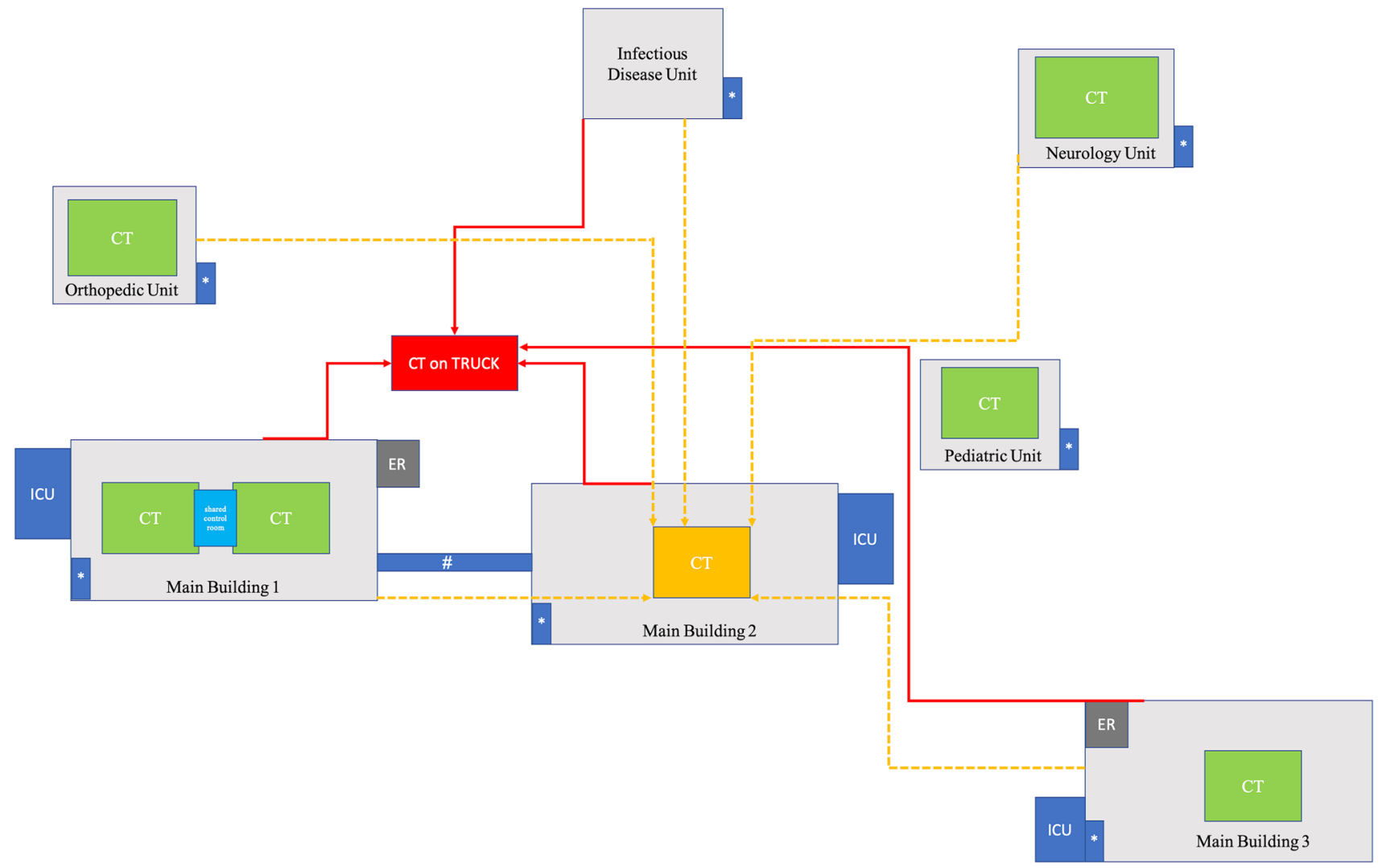

Fig. 1 Graphic representation of the radiological pathway established in our tertiary center to face the COVID-19 outbreak. All patients with a confirmed diagnosis of COVID-19 are scanned in a mobile CT scanner (i.e., represented in red), which is available since the early phase of the outbreak in our region. All suspect COVID-19 patients requiring an urgent $\mathrm{CT}$ are scanned with the device of the academic radiological unit (i.e., represented in orange). All other CTs of the hospital (i.e., represented in green) are used for COVID-19-negative patients. In case of extremely critical patients, the nearest CT room

Author contributions The manuscript has not been published or is considered for publication, elsewhere. All authors have approved the manuscript and have significantly contributed to it.

Funding None of the authors has any financial support.

\section{Compliance with ethical standards}

Conflict of interest None of the authors has any conflict of interest to declare.

Ethical approval All procedures in studies involving human participants were in accordance with the ethical standards of the institutional and/or national research committee and with the 1964 Declaration of Helsinki and its later amendments or comparable ethical standards. is used and then disinfected and locked according to the guidelines. [Only the main buildings of the hospital where COVID-19 are currently admitted and the radiological units are pictured. Other units such as the women care center with patients in need of radiological examinations will follow the same pathway for suspected and confirmed COVID-19 patients and use the nearest CT room for COVID19-negative patients. \#safe transport with Individual Protection Devices; *COVID-19 only elevators. ICU: Intensive Care Unit; CT: Computed Tomography]

Informed consent For this type of study, formal consent is not required.

Consent for publication For this type of study, consent for publication is not required.

\section{References}

1. Grasselli G, Pesenti A, Cecconi M (2020) Critical care utilization for the COVID-19 outbreak in Lombardy, Italy. Early experience and forecast during an emergency response. JAMA. https://doi. org/10.1001/jama.2020.4031

2. He Y, Lin Z, Tang D, Yang Y, Wang T, Yang M (2020) Strategic plan for management of COVID-19 in paediatric haematology and 
oncology departments. Lancet Haematol. https://doi.org/10.1016/ S2352-3026(20)30104-6

3. Giovagnoni A (2020) Facing the COVID-19 emergency: we can and we do. Radiol Med. https://doi.org/10.1007/s11547-02001178-y

4. Parmar HA, Lim TC, Goh JS et al (2004) Providing optimal radiology service in the severe acute respiratory syndrome outbreak: use of mobile CT. AJR Am J Roentgenol 182:57-60

5. ACR Recommendations for the use of Chest Radiography and Computed Tomography (CT) for Suspected COVID-19 Infection (2020) https://www.acr.org/Advocacy-and-Economics/ACR-Posit ion-Statements/Recommendations-for-Chest-Radiography-andCT-for-Suspected-COVID19-Infection. Accessed 7 Apr 2020.

6. Agostini A, Floridi C, Borgheresi A et al (2020) Proposal of a low-dose, long-pitch, dual-source chest CT protocol on thirdgeneration dual-source CT using a tin filter for spectral shaping at $100 \mathrm{kVp}$ for CoronaVirus Disease 2019 (COVID-19) patients: a feasibility study. Radiol Med. https://doi.org/10.1007/s11547-02001179-x

7. ACR COVID-19 Clinical Resources for Radiologists. Clinical https://www.acr.org/Clinical-Resources/COVID-19-RadiologyResources . Accessed 4 May 2020.

8. Mossa-Basha M, Meltzer CC, Kim DC, Tuite MJ, Kolli KP, Tan BS (2020) Department preparedness for COVID-19: radiology scientific expert panel. Radiology 16:200988. https://doi. org/10.1148/radiol.2020200988

Publisher's Note Springer Nature remains neutral with regard to jurisdictional claims in published maps and institutional affiliations. 